\title{
TU/e EmonONEN

\section{Using uterine activity to improve fetal heart rate variability analysis for detection of asphyxia during labor}

\section{Citation for published version (APA):}

Warmerdam, G. J. J., Vullings, R., van Laar, J. O. E. H., van der Hout-van der Jagt, M. B., Bergmans, J. W. M., Schmitt, L., \& Oei, S. G. (2016). Using uterine activity to improve fetal heart rate variability analysis for detection of asphyxia during labor. Physiological Measurement, 37(3), 387-400. https://doi.org/10.1088/0967$3334 / 37 / 3 / 387$

DOI:

10.1088/0967-3334/37/3/387

Document status and date:

Published: 10/02/2016

\section{Document Version:}

Accepted manuscript including changes made at the peer-review stage

\section{Please check the document version of this publication:}

- A submitted manuscript is the version of the article upon submission and before peer-review. There can be important differences between the submitted version and the official published version of record. People interested in the research are advised to contact the author for the final version of the publication, or visit the $\mathrm{DOI}$ to the publisher's website.

- The final author version and the galley proof are versions of the publication after peer review.

- The final published version features the final layout of the paper including the volume, issue and page numbers.

Link to publication

\section{General rights}

Copyright and moral rights for the publications made accessible in the public portal are retained by the authors and/or other copyright owners and it is a condition of accessing publications that users recognise and abide by the legal requirements associated with these rights.

- Users may download and print one copy of any publication from the public portal for the purpose of private study or research.

- You may not further distribute the material or use it for any profit-making activity or commercial gain

- You may freely distribute the URL identifying the publication in the public portal.

If the publication is distributed under the terms of Article 25fa of the Dutch Copyright Act, indicated by the "Taverne" license above, please follow below link for the End User Agreement:

www.tue.nl/taverne

Take down policy

If you believe that this document breaches copyright please contact us at:

openaccess@tue.nl

providing details and we will investigate your claim. 


\title{
Using uterine activity to improve fetal heart rate variability analysis for detection of asphyxia during labor
}

\author{
G.J.J. Warmerdam ${ }^{1}$, R. Vullings ${ }^{1}$, J.O.E.H. Van Laar $^{2}$, M.B. Van der \\ Hout-Van der Jagt ${ }^{2}$, J.W.M. Bergmans ${ }^{1}$, L. Schmitt ${ }^{3}$, and S.G. Oei ${ }^{2}$ \\ ${ }^{1}$ Faculty of Electrical Engineering, Eindhoven University of Technology, Eindhoven, The \\ Netherlands \\ ${ }^{2}$ Máxima Medical Center, Veldhoven, The Netherlands. \\ ${ }^{3}$ Philips Research, Eindhoven, The Netherlands. \\ E-mail: $g \cdot j \cdot j \cdot$ warmerdam@tue.nl
}

November 2015

\begin{abstract}
During labor, uterine contractions can cause temporary oxygen deficiency for the fetus. In case of severe and prolonged oxygen deficiency this can lead to asphyxia. The currently used technique for detection of asphyxia, cardiotocography (CTG), suffers from a low specificity. Recent studies suggest that analysis of fetal heart rate variability (HRV) in addition to CTG can provide information on fetal distress. However, interpretation of fetal HRV during labor is difficult due to the influence of uterine contractions on fetal HRV. The aim of this study is therefore to investigate whether HRV features differ during contraction and rest periods, and whether these differences can improve the detection of asphyxia. To this end, a case-control study was performed, using 14 cases with asphyxia that were matched with 14 healthy fetuses. We did not find significant differences for individual HRV features when calculated over the fetal heart rate without separating contractions and rest periods ( $p>0.30$ for all HRV features). Separating contractions from rest periods did result in a significant difference. In particular the ratio between HRV features calculated during and outside contractions can improve discrimination between fetuses with and without asphyxia ( $p<0.04$ for three out of four ratio HRV features that were studied in this paper).
\end{abstract}

Keywords: Intrapartum monitoring, fetal heart rate variability, asphyxia, uterine contractions Submitted to: Physiol. Meas.

\section{Introduction}

During labor, uterine contractions can cause temporary oxygen deficiency for the fetus. Although a fetus is generally well-equipped to handle brief periods of oxygen deficiency, if oxygen deficiency is severe and prolonged it can result in asphyxia which is associated with neonatal morbidity and mortality (Low et al., 1997). It is therefore important that clinicians can timely intervene before asphyxia develops. 
Currently, cardiotocography (CTG) is the most often used method for detection of asphyxia. CTG simultaneously records the fetal heart rate (FHR) and uterine activity (UA). The CTG is interpreted visually, based on standardized guidelines (FIGO, 1995). Despite these guidelines, CTG interpretation has a high inter- and intra-observer variability (Blix et al., 2003). Furthermore, the specificity of CTG is low, leading to a high false alarm rate (Alfirevic et al., 2006). To reduce the number of unnecessary operative deliveries, additional information is required when the CTG is abnormal.

Recent studies have shown interest in analysis of fetal heart rate variability (HRV) to aid in the detection of asphyxia (van Laar et al., 2010, 2011; Gonçalves et al., 2006; Spilka et al., 2012; Georgieva et al., 2013; Abry et al., 2013). Because variations in the heart rate are regulated by the autonomic nervous system (ANS), various HRV features have been developed that attempt to quantify different aspects of the cardiac autonomic regulation (Camm et al., 1996). Most of these features have been developed for adults, but they might also provide diagnostic information about fetal distress. Note that HRV features are usually defined for the analysis of interbeat interval (R-R interval) signals and HRV features should thus not be applied directly to the FHR signal in beats per minute (BPM). Instead FHR should be recalculated to $\mathrm{R}-\mathrm{R}$ intervals first.

Reviews of HRV features can be found in Van Laar et al. (2008), Voss et al. (2009), Bravi et al. (2011), and Spilka et al. (2012). Features describing the statistical properties of a FHR signal have been used in Magenes et al. (2000) and Gonçalves et al. (2006) for detection of fetal asphyxia. In van Laar et al. (2010), spectral analysis was used to examine the effect of fetal asphyxia on the regulation of the sympathetic and parasympathetic branch of the ANS. Since the sympathetic and parasympathetic nervous system control the heart rate in different frequency bands, spectral analysis is able to separate regulation by these two branches (Akselrod et al., 1985). van Laar et al. (2010) showed that for fetuses with asphyxia normalized low frequency power increased while normalized high frequency power decreased, indicating a predominance of sympathetic regulation.

Spectral analysis is based on a linear model to describe the relation between ANS regulation and variations in the heart rate. Besides spectral analysis, other HRV features have been developed that quantify variations that cannot be explained by this linear model. In Li et al. (2005), Gonçalves et al. (2006), Ferrario et al. (2006), and Signorini et al. (2014), FHR signals were analyzed using HRV features that quantify the complexity in a time series. Furthermore, fractal analysis has also been employed to describe the FHR signal during labor (Abry et al., 2013; Chudacek et al., 2014).

To improve the predictive capacity of HRV features, several studies have combined multiple HRV features by using classifiers such as support vector machines (Spilka et al., 2012; Georgoulas et al., 2006) or neural networks (Georgieva et al., 2013).

Despite these efforts, it remains difficult to interpret HRV features calculated from a FHR signal during labor, since most HRV features have been developed for adults. Unlike for adults, changes in the fetal cardiovascular system during labor cannot be controlled and they are strongly influenced by uterine contractions. There are several ways by which contractions can influence the fetal cardiovascular system (Sundström et al., 2000). The rise in external 
pressure can directly change the fetal blood pressure, which activates a baroreceptor reflex. Contractions can also indirectly influence the fetal cardiovascular system, by blocking the oxygen supply to the fetus (e.g. due to umbilical cord occlusion or reduced maternal blood flow to the placenta), activating a chemoreceptor reflex. After a contraction, some time is needed for the fetal blood gases to recover. In addition, labor can be divided into different stages and the influence of contractions on the fetus changes over time. The first stage is a period of progressive cervical dilation, followed by the second stage of active pushing where the fetal head descends into the birth canal. As a result of this uncontrolled environment, HRV features are often calculated over highly non-stationary FHR signals.

This is also indicated by Romano et al. (2006) and Cesarelli et al. (2010), who showed that for healthy fetuses spectral power is significantly higher during contractions as compared to rest periods (i.e. the periods in between contractions). The increase in spectral power indicates a capability of the ANS to react to uterine contractions. Calculating HRV features over the entire FHR signal, without consideration of contractions, averages this effect over the rest periods.

The aim of this study was therefore to examine whether separating contractions from rest periods can improve HRV analysis for the detection of asphyxia during labor. To this end, a case-control study was used to examine the value of several conventional HRV features in discriminating between fetuses with and without asphyxia.

\section{Methods}

\subsection{Data acquisition}

Data for this study was obtained at the Máxima Medical Center in Veldhoven and the University Medical Center in Utrecht over the period 2001 to 2008. All registrations were recorded on a Neoventa STAN ${ }^{\circledR}$ system. We used beat-to-beat FHR signals that were recorded using a scalp electrode. The UA was measured either via a tocodynamometer or via an intra-uterine pressure catheter (IUPC).

Registrations were included from healthy mothers who had an uncomplicated pregnancy of at least 36 weeks of gestation and did not use any medication. Pregnancies complicated by intra-uterine growth restriction or fetal congenital anomalies were excluded. Furthermore, we only included registrations with at least 15 minutes of FHR signal during the second stage of labor. Contractions were annotated by a clinical expert based on the UA trace, who was blinded to the FHR signal and any other patient or medical information, including the fetal outcome. Only registrations with good quality UA trace were used, defined as registrations where the expert was able to distinguish the contractions.

Fetal outcome was based on the umbilical arterial acid-base status; a pH above 7.20 was defined as healthy, and a $\mathrm{pH}$ below 7.05 and base excess below -12 as asphyxia (Sundström et al., 2000; Westerhuis et al., 2010). Out of the 1232 registrations, 14 cases of fetuses with asphyxia remained for analysis, that were matched with the registration of 14 healthy fetuses that were randomly selected from the available data. Besides the inclusion criteria mentioned 
Table 1. Clinical characteristics of included fetuses.

\begin{tabular}{lcc}
\hline Clinical features & $\begin{array}{c}\text { fetuses without asphyxia } \\
\text { (cases } \mathrm{n}=14)\end{array}$ & $\begin{array}{c}\text { fetuses with asphyxia } \\
\text { (cases } \mathrm{n}=14)\end{array}$ \\
\hline Gestational age (days) & $284 \pm 10$ & $284 \pm 9$ \\
Birth weight (g) & $3773 \pm 587$ & $3601 \pm 442$ \\
1-minute Apgar score & $9 \pm 1$ & $7 \pm 2$ \\
5-minute Apgar score & $10 \pm 0$ & $8 \pm 2$ \\
Cord arterial pH & $7.26 \pm 0.06$ & $6.99 \pm 0.06$ \\
Cord arterial base excess (mmol/l) & $-5 \pm 2$ & $-17 \pm 3$ \\
Length second stage of labor (min) & $58 \pm 27$ & $71 \pm 49$ \\
Start before birth (min) & $17 \pm 3$ & $25 \pm 11$ \\
\hline
\end{tabular}

Values are expressed as mean and standard deviation.

in the previous paragraph, no further criteria were used to match the two populations. The main clinical characteristics of the included fetuses is shown in table 1.

\subsection{Signal processing}

HRV features were calculated on R-R interval signals that were extracted from Neoventa STAN $^{\circledR}$ recordings. Since beat-to-beat R-R intervals can only be determined when a heartbeat occurs, the R-R intervals are not equidistantly sampled. To obtain an equidistantly sampled signal, a sample and hold technique was used (Peters et al., 2011). To obtain a smooth function, the resulting signal was convoluted with a square function of width $0.5 \mathrm{~s}$ and sampled at a rate of $4 \mathrm{~Hz}$.

When the difference between two successive R-R intervals was more than 25 BPM or more than $200 \mathrm{~ms}$, both R-R intervals were considered as artifacts. The reason we also used $200 \mathrm{~ms}$ as criterion is that, in case of very low heart rate, it can occur that the difference between successive beats is more than $200 \mathrm{~ms}$ but less than 25 BPM. Artifacts were corrected by linear interpolation from the previous correct R-R interval to a new segment of 5 consecutive R-R intervals with less than 10 BPM difference between them (Georgoulas et al., 2006). For each registration, we analyzed the 15 minutes closest to birth, where there was no loss of UA signal and less than $20 \%$ interpolation of the FHR signal.

\subsection{HRV features}

For each 15 minute FHR signal, conventional HRV features were calculated that describe different properties of the heart rate. Since there are several reviews on HRV features in the literature (e.g. Voss et al., 2009; Bravi et al., 2011; Spilka et al., 2012), this section only briefly discusses the underlying physiological model and implementation of the HRV features used in this study.

2.3.1. Statistical features The standard deviation $(S D)$ and the root mean square of successive differences $(R M S S D)$ were calculated as statistical features. $S D$ is a measure for 
the overall variability in the heart rate and it reflects both sympathetic and parasympathetic regulation. Since $R M S S D$ is sensitive to beat-to-beat variations, it mainly reflects parasympathetic regulation (Schneider et al., 2009).

Let $x[n]$ be the resampled FHR signal for $n=1, \ldots N$, with $N$ the length of the signal. Then $S D$ and $R M S S D$ are defined as

$$
\begin{aligned}
S D & =\sqrt{\frac{1}{N-1} \sum_{n=1}^{N}(x[n]-\bar{x})^{2}} \\
R M S S D & =\sqrt{\frac{1}{N-1} \sum_{n=2}^{N}(x[n]-x[n-1])^{2}},
\end{aligned}
$$

with $\bar{x}$ the mean heart rate.

2.3.2. Frequency domain features Spectral analysis accounts for correlation in a signal. The use of spectral analysis to analyze the heart rate is based on the observation of periodic variations in the heart rate that can be related to different regulatory mechanisms of cardiovascular control (Akselrod et al., 1985). Since regulation of the sympathetic nervous system is slow compared to regulation of the parasympathetic nervous system, low frequency oscillations are related to both sympathetic and parasympathetic regulation, and high frequency oscillations are only related to parasympathetic regulation.

Spectral analysis was performed by the Continuous Wavelet Transform (CWT) with a fifth order symlet wavelet (Peters et al., 2011). In contrast to the traditional Fourier Transform, CWT enables a simultaneous time and frequency analysis, making CWT suitable for analysis of the non-stationary frequency content of a FHR signal. Since in this study we are only interested in the average spectral power, results of the CWT are comparable to other approaches for spectral analysis that account for non-stationarity in a signal, such as the Short Term Fourier Transform (STFT) used by van Laar et al. (2010) or the Welch method used by Gonçalves et al. (2006). However, unlike STFT and Welch method, CWT allows for a multiresolution time frequency analysis. Since artefact correction in the FHR signal mainly affects the higher frequencies, having a higher time resolution for these frequencies will reduce the influence of artefact correction (Peters et al., 2011).

The CWT compares the FHR signal $(x[n])$ to an analyzing wavelet function $\psi$. A wavelet is defined as a mother wavelet function $\psi[n]$, with a family of scaled and time-shifted daughter wavelets $\psi\left[\frac{n-\tau}{s}\right]$. The CWT coefficients $W[s, \tau]$ can be obtained by continuously varying the scale parameter $s$ and the position parameter $\tau$. For real discrete signals, as is the case for the FHR signal, $W[s, \tau]$ can be calculated according to

$$
W[s, \tau]=\frac{1}{\sqrt{s}} \sum_{n=1}^{N} x[n] \psi\left[\frac{n-\tau}{s}\right] .
$$

To correct for the convolution of the beat-to-beat FHR signal with a square function that was used for smoothing (as explained in section 2.2), $W[s, \tau]$ is first divided by the coefficients of the wavelet transform of this square function. From the corrected wavelet coefficients 
$(\tilde{W}[s, \tau])$ the power at each time instant at a certain scale is calculated as

$$
P[s, \tau]=\frac{1}{C_{g}}\left(\frac{\tilde{W}[s, \tau]}{s}\right)^{2},
$$

where $C_{g}$ is the admissibility constant. Since each daughter wavelet is related to a certain frequency, the total power in a frequency band is then calculated by integrating $P[s, \tau]$ over the scales within that frequency band and by averaging over time. Note that unlike the discrete wavelet transform, the CWT is not limited to a dyadic decomposition of a signal in the timefrequency plane (Addison, 2002). The scale is a continuous parameter and can be selected such that it corresponds to a frequency within a certain frequency band.

The low frequency band was set to $0.04-0.15 \mathrm{~Hz}$ and the high frequency band to 0.4$1.5 \mathrm{~Hz}$ (van Laar et al., 2010). Scales corresponding to the frequencies between these bands $(0.15-0.4 \mathrm{~Hz})$ were discarded. Besides absolute low frequency power $(L F)$ and high frequency power $(H F)$, also normalized $L F\left(L F_{n}\right)$ and normalized $H F\left(H F_{n}\right)$ power are calculated by dividing $L F$ and $H F$ by the total power (TP, 0.04-1.5 Hz) (van Laar et al., 2010).

The minimum length of FHR signal that is required to calculate $L F$ (and $T P$ ) is determined by the largest wavelet of the LF band. Therefore, a segment of at least 50 seconds is required to reliably calculate $L F$ (Peters et al., 2008). Similarly, calculation of normalized frequency powers $\left(L F_{n}\right.$ and $\left.H F_{n}\right)$ also requires 50 seconds.

\subsubsection{Complexity features Although spectral analysis provides information about regulation} mechanisms of the cardiovascular system, variations in the heart rate often cannot be explained by spectral analysis alone. Since many factors can cause blood pressure changes (e.g. changes in external pressure or through chemoreceptor reflex), blood pressure often shows complicated and irregular fluctuations. Because adjusting the heart rate is one of the main tools of the ANS to stabilize these blood pressure fluctuations, also the heart rate can show complicated and irregular fluctuations. The occurrence of irregularities in the heart rate is therefore indicative of healthy blood pressure control by the ANS.

A measure that is often used to quantify the complexity of very short signals in terms of randomness is Sample Entropy (SampEn) (Richman and Moorman, 2000). SampEn estimates the conditional probability that when patterns are similar for length $m-1$, they will also be similar for length $m$. This probability is small in case the heart rate is complex and large in case the heart rate is not complex.

To calculate SampEn, the FHR signal is divided vectors $\mathbf{u}$ of length $m$

$$
\mathbf{u}[i]=\{x[i], x[i+1], \ldots, x[i+m-1]\} .
$$

Two vectors $\mathbf{u}[i]$ and $\mathbf{u}[j]$ are similar if the maximum distance between the elements of $\mathbf{u}[i]$ and $\mathbf{u}[j]$ is less than tolerance parameter $r$. Let us define $C_{i}^{m}[r]$ as the number of vectors $\mathbf{u}[j]$ that are similar to $\mathbf{u}[i]$ (for $j \neq i$ ), normalized by the total number vectors of length $m$. The probability $\left(\Phi^{m}\right)$ of finding similar vectors of length $m$ is then calculated as the average of $C_{i}^{m}[r]$

$$
\Phi^{m}[r]=(N-m+1)^{-1} \sum_{i=1}^{N-m+1} C_{i}^{m}[r] .
$$


SampEn is the natural logarithm of the conditional probability in the limit $N \rightarrow \infty$

$$
\operatorname{SampEn}[m, r]=\lim _{N \rightarrow \infty}-\ln \frac{\Phi^{m+1}[r]}{\Phi^{m}[r]} .
$$

Similar to Gonçalves et al. (2006), Ferrario et al. (2006), and Spilka et al. (2012), the embedding dimension $m$ was set to $m=2$ and the tolerance to $r=0.2 S D$. For finite time series, SampEn can be approximated reliably with $N>100$ samples (Richman and Moorman, 2000).

2.3.4. Fractal features Besides examining how the heart rate is controlled at certain specific time scales (e.g. LF and HF band for spectral analysis), Goldberger et al. (2002) observed that, for adults, fluctuations in the heart rate show self-similarity on different time scales. In this context, self-similarity means that structure of the heart rate on long time scales is resembled at shorter time scales. Fractal analysis describes the scaling properties of the heart rate. In Abry et al. (2013), fractal analysis was used to investigate the scaling properties of the FHR during labor.

In our study, the variability at different time scales is described by the scaling exponent $\alpha$, which is obtained by Detrended Fluctuation Analysis (DFA) (Peng et al., 1995; Ihlen, 2012). To calculate $\alpha$, first an integrated version of the original time series is obtained:

$$
y[k]=\sum_{n=1}^{k}(x[n]-\bar{x}) .
$$

Next, $y[k]$ is divided into equally spaced segments of length $a$ and the linear trend of each segment $\left(y_{a}[k]\right)$ is determined. The characteristic fluctuation $F$ at each scale $a$ is computed as the root mean square (RMS) deviation between $y[k]$ and its trend:

$$
F[a]=\sqrt{\frac{1}{N} \sum_{k=1}^{N}\left(y[k]-y_{a}[k]\right)^{2} .}
$$

If a linear relation exists between $\log (F[a])$ and $\log (a)$, the heart rate is said to show selfsimilarity, such that variability at long time scales is related to variability at shorter time scales in a power-law fashion $\left(F[a] \sim a^{\alpha}\right)$. The slope $\alpha$ of the relation between $\log (F[a])$ and $\log (a)$ characterizes the type of the time series (e.g. $\alpha=0.5$ for white noise and $\alpha=1.5$ for Brownian noise). Also note that $\alpha$ is related to the Hurst exponent used in Abry et al. (2013) as $H=\alpha-1$ (Eke et al., 2002).

Similar to Abry et al. (2013), $\alpha$ was computed over the scales ranging from 1 to 60 seconds, which corresponds to the physiological range of the baroreflex.

\subsection{HRV analysis during contractions and rest periods}

For calculation of HRV features during contractions and rest periods separately, the expert annotations of the contractions were used. To calculate HRV features during contractions, values based on R-R intervals measured during rest periods were excluded. Similarly, to calculate HRV features during rest periods, values based on R-R intervals measured during 
Table 2. HRV analysis over the entire FHR signal.

\begin{tabular}{lccc}
\hline HRV features & fetuses without asphyxia & fetuses with asphyxia & Significance (p-value) \\
\hline$S D$ & $78[56-116]$ & $96[52-122]$ & 0.80 \\
$R M S S D$ & $7.7[4.0-8.9]$ & $6.7[4.8-7.8]$ & 0.37 \\
$L F$ & $817[202-1147]$ & $478[201-1290]$ & 0.45 \\
$H F$ & $24[11-54]$ & $20[12-31]$ & 0.60 \\
$L F_{n}$ & $0.78[0.73-0.83]$ & $0.80[0.73-0.85]$ & 0.32 \\
$H F_{n}$ & $0.06[0.05-0.10]$ & $0.06[0.03-0.08]$ & 0.48 \\
SampEn & $0.17[0.09-0.24]$ & $0.16[0.11-0.22]$ & 0.98 \\
$\alpha$ & $1.40[1.21-1.55]$ & $1.43[1.37-1.56]$ & 0.37 \\
\hline
\end{tabular}

Values are expressed as median [inter quartile range].

Table 3. HRV analysis during contractions.

\begin{tabular}{lccc}
\hline HRV features & fetuses without asphyxia & fetuses with asphyxia & Significance (p-value) \\
\hline$S D$ & $78[55-123]$ & $72[49-121]$ & 0.54 \\
$R M S S D$ & $9.5[4.6-11.6]$ & $7.2[3.9-8.7]$ & 0.16 \\
$H F$ & $38[13-72]$ & $27[9-47]$ & 0.30 \\
SampEn & $0.22[0.16-0.30]$ & $0.20[0.13-0.26]$ & 0.30 \\
\hline
\end{tabular}

Values are expressed as median [inter quartile range].

contractions were excluded. In our study, we also considered the ratio between HRV features calculated during contractions and rest periods.

It is important to note that in the second stage of labor the duration of contractions and rest periods is often less than one minute. Since computation of $L F, L F_{n}, H F_{n}$, and $\alpha$ requires approximately one minute of consecutive FHR signal, we could not extract these HRV features during contractions and rest periods separately.

Our study of HRV features during contractions and rest periods was thus limited to $S D$, RMSSD, HF, and SampEn. Besides calculating these HRV features during contractions and during rest periods, we also examined the ratio between HRV features calculated during contractions and rest periods.

\subsection{Statistical methods}

A Wilcoxon ranksum test was used to determine for each HRV feature whether the median of the distribution of the healthy group was significantly different from the median of the distribution of the group with asphyxia (Gibbons and Chakraborti, 2011). Statistical significance was set at a $p$ value of less than 0.05 .

\section{Results}

The HRV features discussed in the previous section were calculated from the 15 minute FHR signals. Results are shown in tables 2-5 as median and inter quartile range. An example of a 
Table 4. HRV analysis during rest periods.

\begin{tabular}{lccc}
\hline HRV features & fetuses without asphyxia & fetuses with asphyxia & Significance (p-value) \\
\hline$S D$ & $54[39-80]$ & $83[50-117]$ & 0.22 \\
$R M S S D$ & $4.3[3.0-7.4]$ & $5.7[4.3-6.3]$ & 0.60 \\
$H F$ & $11[4-29]$ & $17[8-21]$ & 0.66 \\
SampEn & $0.11[0.07-0.18]$ & $0.11[0.08-0.16]$ & 0.57 \\
\hline
\end{tabular}

Values are expressed as median [inter quartile range].

Table 5. HRV analysis: ratio between contractions and rest periods.

\begin{tabular}{lccc}
\hline HRV features & fetuses without asphyxia & fetuses with asphyxia & Significance (p-value) \\
\hline $\boldsymbol{S D}$ & $\mathbf{1 . 6}[\mathbf{1 . 1}-\mathbf{2 . 0}]$ & $\mathbf{1 . 0}[\mathbf{0 . 9}-\mathbf{1 . 2}]$ & $\mathbf{0 . 0 1}$ \\
$\boldsymbol{R M S S D}$ & $\mathbf{1 . 7}[\mathbf{1 . 4 - 2 . 3}]$ & $\mathbf{1 . 4}[\mathbf{1 . 1 - 1 . 6}$ & $\mathbf{0 . 0 2}$ \\
HF & $3.0[1.8-5.9]$ & $2.1[1.3-2.6]$ & 0.16 \\
SampEn & $\mathbf{1 . 8}[\mathbf{1 . 5 - 3 . 0}]$ & $\mathbf{1 . 4}[\mathbf{1 . 1}-\mathbf{1 . 7}]$ & $\mathbf{0 . 0 3}$ \\
\hline
\end{tabular}

Values are expressed as median [inter quartile range].
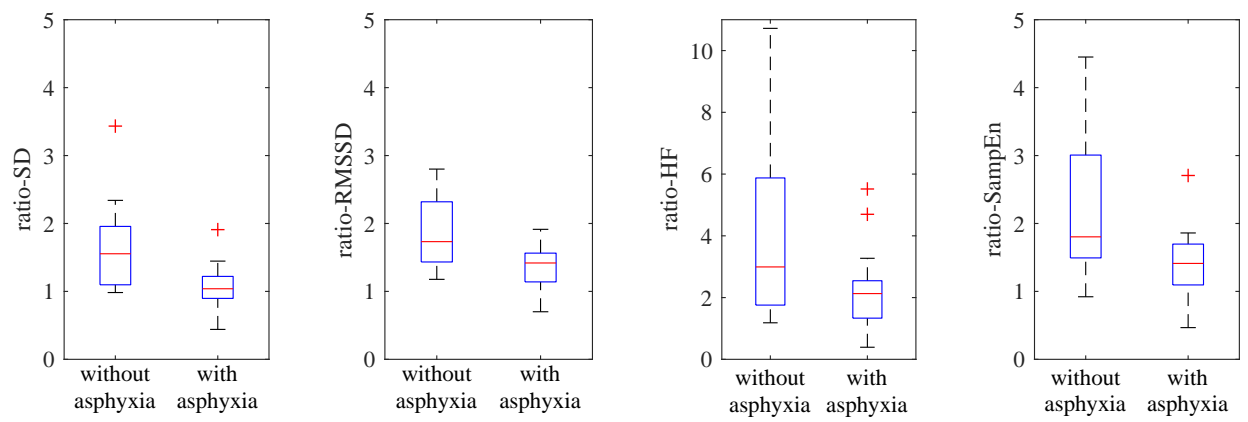

Figure 1. Boxplots of the ratio between HRV features calculated during contractions and rest periods. The central mark and the edges of the box indicate the median and inter quartile range. The whiskers correspond to the most extreme values that are not considered outliers (where outliers are defined as $\pm 2.7 \sigma$, with $\sigma$ the standard deviation).

CTG registration of a healthy fetus and that of a fetus with asphyxia is displayed in figure 2 .

Table 2 shows the results for HRV features that were calculated over the entire FHR signal. No significant difference in median was found between the group with and without asphyxia for any of the individual HRV features. HRV features calculated during contractions are compared for fetuses with and without asphyxia in table 3, and HRV features calculated during rest are compared for fetuses with and without asphyxia in table 4. No significant difference was found between fetuses with and without asphyxia either during contractions or during rest periods. Finally, table 5 shows the results of the ratio of HRV features calculated during contractions and HRV features calculated during rest periods. For $S D(p=0.01)$, $R M S S D(p=0.02)$, and SampEn $(p=0.03)$ the ratio was significantly lower for the group with asphyxia compared with the healthy group. Although the median ratio for $H F$ was also lower for the group with asphyxia, this difference was not significant. Complementary to 


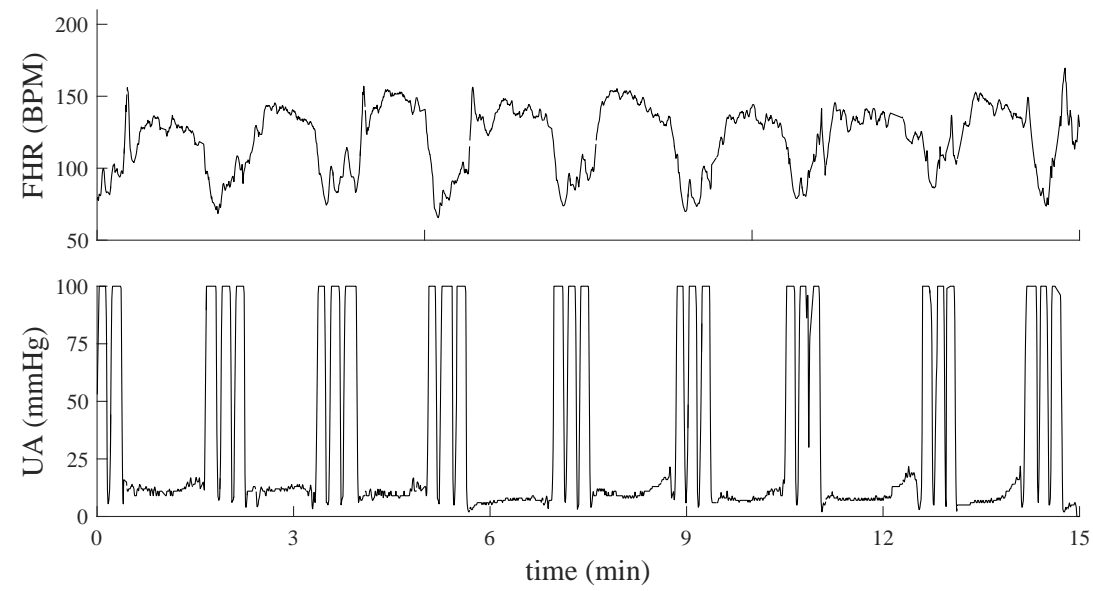

(a)
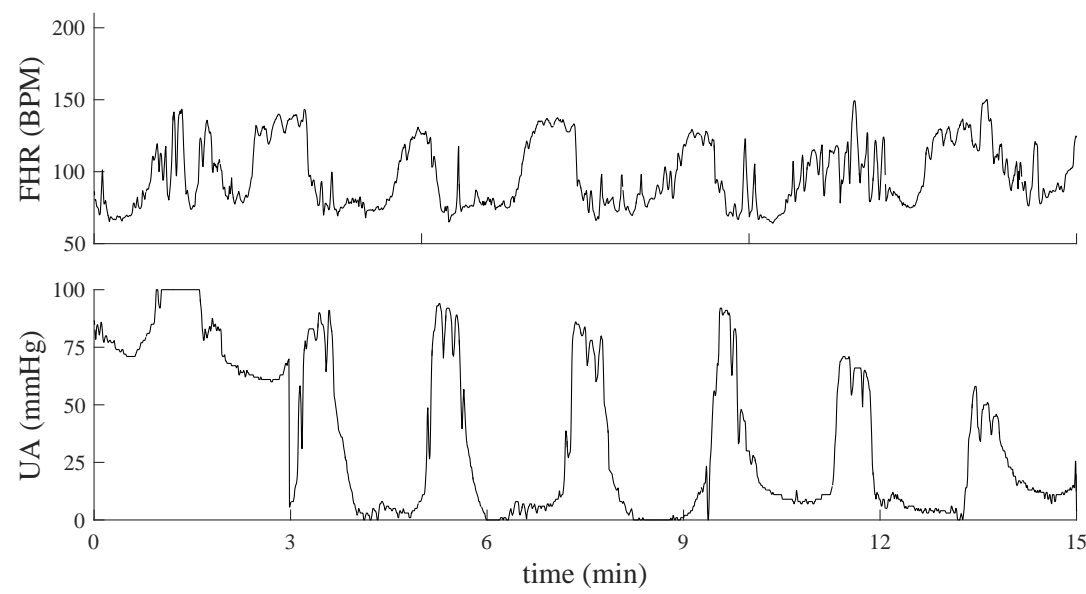

(b)

Figure 2. Example of a FHR signal of a healthy fetus (a) and a fetus with asphyxia (b). For the healthy fetus, the ratios between HRV features during contractions and rest periods are: $S D=3.4, R M S S D=2.8, H F=10.5$, and $S a m p E n=4.5$. For the fetus with asphyxia, the ratios are: $S D=1.0, R M S S D=1.6, H F=2.1$, and $\operatorname{SampEn}=1.7$.

results in table 5, the distributions of the ratio of HRV features are shown as boxplots in figure 1.

\section{Discussion}

There are many factors that determine the response of the fetus to a temporal loss of oxygen caused by uterine contractions. Therefore, CTG registrations often display a large variety of complicated patterns, making it difficult to interpret them. Automated analysis of fetal HRV attempts to support clinicians in their interpretation by providing additional quantitative information on the cardiovascular system. However, existing HRV features have been mainly developed for adults and do not account for the strong influence of uterine contractions on the cardiovascular control. When calculating these HRV features over the entire FHR signal, the 
effect of contractions is averaged with rest periods. The goal of this study was to show that separating contractions from rest periods improves HRV analysis for the detection of fetal asphyxia.

Despite the relatively large number of available registrations, only 14 cases with $\mathrm{pH}$ below 7.05 could be included for the analysis. This is partly due to the low incidence of asphyxia (between 0.6\% (Heintz et al., 2008) and 3.5\% (d'Aloja et al., 2009)), but also because of the strict inclusion criteria we used. We required a good quality UA trace, so that the expert could clearly identify contractions. In clinical practice, however, it frequently occurs that the UA trace is of poor quality (Bakker et al., 2008). A potential solution might be the use of electrohysterography for the registration of UA (Euliano et al., 2013). Note that in our dataset the number of registrations that measured UA with tocodynamometer or with IUPC was the same for both the healthy group and the group with asphyxia (10 with tocodynamometer and 4 with IUPC). Since we only included registrations with good quality UA trace, we expect that the use of tocodynamometer or IUPC did not influence our results.

For some recordings we were unable to analyze the final minutes before birth, either due to loss of FHR signal or insufficient quality of UA trace (table 1). As a result, segments for the healthy group were selected closer to birth than for the group with asphyxia. Because it has been reported that, in absence of sudden catastrophic events, asphyxia in term fetuses develops within a period of at least 90 minutes (Fleischer et al., 1982), we can assume that fetuses in the group with asphyxia were already under more stressful conditions than fetuses in the healthy group. We expect the effect of asphyxia to increase as labor progresses and differences between healthy fetuses and fetuses with asphyxia to be even more pronounced during the final minutes before birth.

\subsection{HRV features over the entire FHR signal}

We did not find a significant difference between the healthy group and the group with asphyxia for individual HRV features when calculated over the entire FHR signal (table 2). This could partly be explained by the small number of recordings and large inter-patient variability of the HRV features.

For absolute $L F$ and $H F$, our results are similar to the findings in Gonçalves et al. (2006) and van Laar et al. (2010), despite the different method they used for spectral analysis (Welch method and STFT respectively, versus CWT used in this study). Although in our study normalized $L F$ was higher for the group with asphyxia, in line with van Laar et al. (2010), this difference was not significant. Furthermore, in contrast to the findings in Gonçalves et al. (2006), SampEn was not statistically different. These differences in results obtained in our study and the studies of Gonçalves et al. (2006) and van Laar et al. (2010), might be because Gonçalves et al. (2006) and van Laar et al. (2010) calculated the HRV features over short segments of FHR signal (5 minutes). As discussed in Gonçalves et al. (2006), HRV analysis could be more efficient when performed on shorter segments. However, we used longer segments because in shorter segments a bias could be introduced due to an unbalance in the presence of contractions and rest periods within the segment. The use of shorter segments 
can therefore lead to large intra-patient variability of HRV features over time.

In our study also no difference was found between healthy fetuses and fetuses with asphyxia for the scaling exponent $\alpha$. Recently Chudacek et al. (2014) showed promising results calculating the scaling exponent using a wavelet based scattering transform. This might improve the fractal analysis.

\subsection{HRV features during contractions and rest periods}

Similar to Romano et al. (2006) and Cesarelli et al. (2010), we found that for healthy fetuses $H F$ was significantly increased during contractions with respect to rest periods $(p=0.01)$. This result has not been shown explicitly, but can be seen by comparing the left columns of tables 3 and 4. The increase in HRV features during contractions relative to the HRV features during rest periods indicates that a stronger cardiovascular control is required to stabilize the blood pressure during contractions. The rise in external pressure due to contractions can activate a combination of baroreceptor and chemoreceptor reflexes, leading to relatively high HRV during contractions. This finding was confirmed by the observed increase in RMSSD $(p<0.01)$ and SampEn $(p<0.01)$. In contrast to the results of the healthy group, for the group with asphyxia HRV features were not significantly different during contractions with respect to rest periods (all $p>0.12$, obtained by comparing the right columns of table 3 and 4).

Despite this increase in HRV features during contractions for the healthy group, no significant difference was found when comparing HRV features of the healthy group to the group without asphyxia either during contractions or during rest periods, as shown in tables 3 and 4 respectively. A possible reason why no significant difference was observed between the two groups (either during contractions or during rest periods) might be the large inter-patient variability in obtained values for the HRV features and the relatively small dataset.

\subsection{HRV features: ratio between contractions and rest periods}

When considering the ratio between HRV features calculated during contractions and rest periods, $S D, R M S S D$, and SampEn were all significantly lower for the group with asphyxia than for the healthy group (table 5). Also the ratio for $H F$ was lower (as can be seen in figure 1 ), but this was not significant due to the large spread in the distribution.

For healthy fetuses, a high ratio of HRV features during contractions and rest periods might indicate a response of the fetal ANS to stabilize the cardiovascular system. On the one hand, during contractions HRV features are high because the fetus needs to adapt its cardiovascular system to changes in external pressure (Romano et al., 2006; Cesarelli et al., 2010). On the other hand, during rest periods little cardiovascular control is required because in the second stage of labor fetal movement is reduced (Richardson et al., 1979). Furthermore, after a contraction has ended, a healthy fetus has not suffered much from indirect effect of contractions on the cardiovascular system (i.e. umbilical cord occlusion or reduced maternal blood flow). Therefore, healthy fetuses quickly recover from contractions and HRV features will be low during rest periods. As a result, a high ratio is observed for HRV features during 
contractions and rest periods. Note that if a fetus is not compromised by contractions, HRV features will be similar during contractions and rest periods, leading to a low ratio rather than a high ratio. However, the case where a fetus does not suffer at all is relatively simple to recognize by a clinician based on the FHR signal (e.g. when there are no decelerations in the FHR trace).

Unlike healthy fetuses, a fetus with asphyxia is not able to adequately adapt its cardiovascular system during contractions. Therefore, HRV features during contractions are relatively low for fetuses with asphyxia compared to healthy fetuses (although not significant, as shown in table 3). Even after a contraction has ended, the oxygen concentration of the fetal blood is still low due to aforementioned indirect effects of contractions on the fetal cardiovascular system. The fetus thus also needs to adapt its cardiovascular system during rest periods, which can lead to decelerations that last until after contractions or even start after contractions have ended (Sundström et al., 2000). HRV features during rest are thus higher for fetuses with asphyxia compared to healthy fetuses (although not significant, as shown in table 4). As a result, the ratio of HRV features during contractions and rest periods is lower for fetuses with asphyxia than for healthy fetuses.

An example of a registration of a fetus with and without asphyxia is shown in figure 2. For the healthy fetus, decelerations occur on top of contractions and a stable heart rate is observed during rest periods. The ratios of HRV features during contractions and rest periods are therefore high $(S D=3.4, R M S S D=2.8, H F=10.5$, and $\operatorname{SampEn}=4.5)$. For the fetus with asphyxia, decelerations are prolonged and last until after the contractions have ended. The ratios of HRV features during contractions and rest periods are therefore low ( $S D=1.0$, $R M S S D=1.6, H F=2.1$, and $\operatorname{SampEn}=1.7$ ).

\subsection{Future perspective}

Our results are promising and show that distinguishing contractions from rest periods improves HRV analysis for detection of asphyxia. However, we only used a small dataset. To determine the diagnostic value for clinical practice, further study is required using a larger dataset. Besides, we only examined the effect on individual HRV features. In Spilka et al. (2012), Georgoulas et al. (2006), Georgieva et al. (2013), and Xu et al. (2014), it was shown that combinations of multiple HRV features using classifiers can improve the detection rate of fetal asphyxia. It is therefore interesting to combine multiple HRV features calculated over the entire fetal heart signal with HRV features calculated during contractions and rest periods. Furthermore, it might also be interesting to monitor changes in HRV features over the course of labor as was done in van Laar et al. (2010), Chudacek et al. (2014), and Warrick et al. (2010).

\section{Conclusion}

Analysis of fetal HRV provides information on fetal asphyxia. Results in our study suggest that separating contractions and rest periods improves HRV analysis for the detection of 
asphyxia during labor. In particular, using the ratio between HRV features calculated from contractions and rest periods improves the ability of the HRV features to discriminate between healthy fetuses and fetuses with asphyxia. Further studies are required to determine the diagnostic value in clinical practice.

\section{Acknowledgments}

This research was performed within the framework of the IMPULS perinatology program. The authors would like to thank A. Kwee, Department of Obstetrics and Gynaecology, University Medical Center Utrecht, for provision of their STAN registrations.

\section{References}

Abry, P., Roux, S., Chudácek, V., Borgnat, P., Goncalves, P. and Doret, M. (2013). Hurst exponent and intrapartum fetal heart rate: Impact of decelerations, Computer-Based Medical Systems (CBMS), 2013 IEEE 26th International Symposium on, IEEE, pp. 131136.

Addison, P. S. (2002). The illustrated wavelet transform handbook: introductory theory and applications in science, engineering, medicine and finance, $\mathrm{CRC}$ press.

Akselrod, S., Gordon, D., Madwed, J. B., Snidman, N. C., Shannon, D. C. and Cohen, R. J. (1985). Hemodynamic regulation: investigation by spectral analysis., Am J Physiol 249(4 Pt 2): H867-H875.

Alfirevic, Z., Devane, D. and Gyte, G. (2006). Continuous cardiotocography (CTG) as a form of electronic fetal monitoring (EFM) for fetal assessment during labour., Cochrane Database Syst Rev (3): CD006066.

Bakker, P., Zikkenheimer, M. and van Geijn, H. (2008). The quality of intrapartum uterine activity monitoring, Journal of perinatal medicine 36(3): 197-201.

Blix, E., Sviggum, O., Koss, K. and Øian, P. (2003). Inter-observer variation in assessment of 845 labour admission tests: comparison between midwives and obstetricians in the clinical setting and two experts, BJOG: An International Journal of Obstetrics \& Gynaecology 110(1): $1-5$.

Bravi, A., Longtin, A. and Seely, A. (2011). Review and classification of variability analysis techniques with clinical applications, Biomed Eng Online 10(1): 90.

Camm, A., Malik, M., Bigger, J., Günter, B., Cerutti, S. and Choen, R. (1996). Task force of the european society of cardiology and the north american society of pacing and electrophysiology. heart rate variability: standards of measurement, physiological interpretation and clinical use., Circulation 93(5): 1043-1065.

Cesarelli, M., Romano, M., Ruffo, M., Bifulco, P. and Pasquariello, G. (2010). Foetal heart rate variability frequency characteristics with respect to uterine contractions, JBiSE 03(10): 10141021. 
Chudacek, V., Andén, J., Mallat, S., Abry, P. and Doret, M. (2014). Scattering transform for intrapartum fetal heart rate variability fractal analysis: A case-control study, Biomedical Engineering, IEEE Transactions on 61(4): 1100-1108.

d'Aloja, E., Müller, M., Paribello, F., Demontis, R. and Faa, A. (2009). Neonatal asphyxia and forensic medicine, The Journal of Maternal-Fetal \& Neonatal Medicine 22(sup3): 54-56.

Eke, A., Herman, P., Kocsis, L. and Kozak, L. (2002). Fractal characterization of complexity in temporal physiological signals, Physiological measurement 23(1): R1.

Euliano, T., Nguyen, M., Darmanjian, S., McGorray, S., Euliano, N., Onkala, A. and Gregg, A. (2013). Monitoring uterine activity during labor: a comparison of 3 methods, American journal of obstetrics and gynecology 208(1): 66-e1.

Ferrario, M., Signorini, M., Magenes, G. and Cerutti, S. (2006). Comparison of entropy-based regularity estimators: application to the fetal heart rate signal for the identification of fetal distress, Biomedical Engineering, IEEE Transactions on 53(1): 119-125.

FIGO (1995). Intrapartum surveillance: recommendation on current practice and overview of new developments, Inter J Gynecol Obstet 49: 213.

Fleischer, A., Schulman, H., Jagani, N., Mitchell, J. and Randolph, G. (1982). The development of fetal acidosis in the presence of an abnormal fetal heart rate tracing. i. the average for gestational age fetus., American journal of obstetrics and gynecology 144(1): 55-60.

Georgieva, A., Payne, S. J., Moulden, M. and Redman, C. (2013). Artificial neural networks applied to fetal monitoring in labour, Neural Computing and Applications 22(1): 85-93.

Georgoulas, G., Stylios, D. and Groumpos, P. (2006). Predicting the risk of metabolic acidosis for newborns based on fetal heart rate signal classification using support vector machines, Biomedical Engineering, IEEE Transactions on 53(5): 875-884.

Gibbons, J. D. and Chakraborti, S. (2011). Nonparametric statistical inference, Springer.

Goldberger, A., Amaral, L., Hausdorff, J., Ivanov, P., Peng, C. and Stanley, H. (2002). Fractal dynamics in physiology: alterations with disease and aging, Proceedings of the National Academy of Sciences 99(suppl 1): 2466-2472.

Gonçalves, H., Rocha, A., Ayres-de Campos, D. and Bernardes, J. (2006). Linear and nonlinear fetal heart rate analysis of normal and acidemic fetuses in the minutes preceding delivery, Medical and Biological Engineering and Computing 44(10): 847-855.

Heintz, E., Brodtkorb, T., Nelson, N. and Levin, L. (2008). The long-term cost-effectiveness of fetal monitoring during labour: a comparison of cardiotocography complemented with st analysis versus cardiotocography alone, BJOG: An International Journal of Obstetrics \& Gynaecology 115(13): 1676-1687.

Ihlen, E. (2012). Introduction to multifractal detrended fluctuation analysis in matlab, Front. Physio. 3.

Li, X., Zheng, D., Zhou, S., Tang, D., Wang, C. and Wu, G. (2005). Approximate entropy of fetal heart rate variability as a predictor of fetal distress in women at term pregnancy, Acta obstetricia et gynecologica Scandinavica 84(9): 837-843. 
Low, J. A., Lindsay, B. G. and Derrick, E. J. (1997). Threshold of metabolic acidosis associated with newborn complications., Am J Obstet Gynecol 177(6): 1391-1394.

Magenes, G., Signorini, M. and Arduini, D. (2000). Classification of cardiotocographic records by neural networks, Proceedings of the IEEE-INNS-ENNS International Joint Conference on Neural Networks. IJCNN 2000. Neural Computing: New Challenges and Perspectives for the New Millennium .

Peng, C., Havlin, S., Stanley, H. and Goldberger, A. (1995). Quantification of scaling exponents and crossover phenomena in nonstationary heartbeat time series, Chaos: An Interdisciplinary Journal of Nonlinear Science 5(1): 82-87.

Peters, C., Vullings, R., Bergmans, J., Oei, S. and Wijn, P. (2008). The effect of artifact correction on spectral estimates of heart rate variability., Conf Proc IEEE Eng Med Biol Soc 2008: 2669-2672.

Peters, C., Vullings, R., Rooijakkers, M., Bergmans, J., Oei, S. and Wijn, P. (2011). A continuous wavelet transform-based method for time-frequency analysis of artefactcorrected heart rate variability data., Physiol Meas 32(10): 1517-1527.

Richardson, B., Natale, R. and Patrick, J. (1979). Human fetal breathing activity during electively induced labor at term., American journal of obstetrics and gynecology 133(3): 247-255.

Richman, J. and Moorman, J. (2000). Physiological time-series analysis using approximate entropy and sample entropy, American Journal of Physiology-Heart and Circulatory Physiology 278(6): H2039-H2049.

Romano, M., Bifulco, P., Cesarelli, M., Sansone, M. and Bracale, M. (2006). Foetal heart rate power spectrum response to uterine contraction, Medical and Biological Engineering and Computing 44(3): 188-201.

Schneider, U., Schleussner, E., Fiedler, A., Jaekel, S., Liehr, M., Haueisen, J. and Hoyer, D. (2009). Fetal heart rate variability reveals differential dynamics in the intrauterine development of the sympathetic and parasympathetic branches of the autonomic nervous system, Physiological Measurement 30(2): 215226.

Signorini, M., Fanelli, A. and Magenes, G. (2014). Monitoring fetal heart rate during pregnancy: contributions from advanced signal processing and wearable technology, Computational and mathematical methods in medicine 2014.

Spilka, J., Chudáček, V., Kouckỳ, M., Lhotská, L., Huptych, M., Janků, P., Georgoulas, G. and Stylios, C. (2012). Using nonlinear features for fetal heart rate classification, Biomedical Signal Processing and Control 7(4): 350-357.

Sundström, A., Rosén, D. and Rosén, K. (2000). Fetal surveillance, Gothenburg: Neoventa Medical $A B$.

van Laar, J., Peters, C., Houterman, S., Wijn, P., Kwee, A. and Oei, S. (2011). Normalized spectral power of fetal heart rate variability is associated with fetal scalp blood ph., Early Hum Dev 87(4): 259-263. 
van Laar, J., Peters, C., Vullings, R., Houterman, S., Bergmans, J. and Oei, S. G. (2010). Fetal autonomic response to severe acidaemia during labour., BJOG 117(4): 429-437.

Van Laar, J., Porath, M., Peters, C. and Oei, S. (2008). Spectral analysis of fetal heart rate variability for fetal surveillance: review of the literature., Acta Obstet Gynecol Scand 87(3): 300-306.

Voss, A., Schulz, S., Schroeder, R., Baumert, M. and Caminal, P. (2009). Methods derived from nonlinear dynamics for analysing heart rate variability, Philosophical Transactions of the Royal Society of London A: Mathematical, Physical and Engineering Sciences 367(1887): 277-296.

Warrick, P., Hamilton, E., Precup, D. and Kearney, R. (2010). Classification of normal and hypoxic fetuses from systems modeling of intrapartum cardiotocography, Biomedical Engineering, IEEE Transactions on, 57(4): 771-779.

Westerhuis, M., Visser, G., Moons, K., van Beek, E., Benders, M., Bijvoet, S., van Dessel, H., Drogtrop, A., van Geijn, H., Graziosi, G. et al. (2010). Cardiotocography plus st analysis of fetal electrocardiogram compared with cardiotocography only for intrapartum monitoring: a randomized controlled trial, Obstetrics \& Gynecology 115(6): 1173-1180.

Xu, L., Redman, C., Payne, S. and Georgieva, A. (2014). Feature selection using genetic algorithms for fetal heart rate analysis, Physiological Measurement 35(7): 13571371. 